2

3

4

5

6

\title{
Perioperative Outcomes are Adversely Affected by Poor Pretransfer Adherence to Acute Limb Ischemia Practice Guidelines
}

S. Keisin Wang MD, Michael P. Murphy MD, Ashley R. Gutwein MD, Natalie A. Drucker MD, Michael

C. Dalsing MD, Raghu L. Motaganahalli MD, Gary W. Lemmon MD, and A. George Akingba MD PhD

Division of Vascular Surgery, Department of Surgery, Indiana University School of Medicine Indianapolis IN

\section{Corresponding Authors:}

Michael P. Murphy MD

Associate Professor

Department of Surgery - Division of Vascular Surgery

Indiana University School of Medicine

1801 Senate Blvd MPC\# 2-3500, Indianapolis, IN 46202

Phone: (317) 962-0282

Fax: (317) 962-0289

Email: mipmurph@iupui.edu

S. Keisin Wang MD

Integrated Vascular Surgery Research Fellow

Department of Surgery - Division of Vascular Surgery

Indiana University School of Medicine

1801 Senate Blvd MPC\# 2-3500, Indianapolis, IN 46202

Phone: (317) 962-0282

Fax: (317) 962-0289

Email: wangkei@iupui.edu

Presentation: The contents of this manuscript has been accepted for oral presentation to VESS Winter 2018 (Vail, CO).

Word Count: 2337 (Abstract, Background, Methods, Results, Discussion, Conclusion)

Disclosures: The authors have no relevant financial conflicts of interest to disclose.

Acknowledgements: The authors wish to thank Luona Sun and Cynthia Richardson for their assistance in data acquisition.

This is the author's manuscript of the article published in final edited form as:

Wang, S. K., Murphy, M. P., Gutwein, A. R., Drucker, N. A., Dalsing, M. C., Motaganahalli, R. L., ... Akingba, A. G. (2018). Perioperative Outcomes are Adversely Affected by Poor Pretransfer Adherence to Acute Limb Ischemia Practice Guidelines. Annals of Vascular Surgery. https://doi.org/10.1016/j.avsg.2017.11.050 
Abstract

39

\section{Objectives:}

The accepted treatment for acute limb ischemia (ALI) is immediate systemic anticoagulation and timely reperfusion to restore blood flow. In this study, we describe the retrospective assessment of pretransfer management decisions by referring hospitals to an academic tertiary care facility and its impact on perioperative adverse events.

\section{Methods:}

A retrospective analysis of ALI patients transferred to us via our Level I Vascular Emergency program from 2010 to 2013 was performed. Patient demographics, comorbidities, Rutherford ischemia classification, time to anticoagulation, and time to reperfusion were tabulated and analyzed for correlation to incidence of major adverse limb events (MALE), mortality, and bypass patency in the perioperative period (30-day postoperative). All time intervals were calculated from the onset of symptoms and categorized into three subcohorts ( $<6 \mathrm{hrs}, 6-48 \mathrm{hrs}$, and $>48 \mathrm{hrs})$.

\section{Results:}

Eighty-seven patients with an average age of $64.0( \pm 16.2)$ years presented to outlying hospitals and was transferred to us with lower extremity ALI. The mean delay from symptom onset to initial referring physician evaluation was $18.3 \mathrm{hrs}$. At that time of evaluation, 53.8\% had Rutherford class IIA ischemia and 36.3\% had class IIB ischemia. Seventy-six (87.4\%) patients were started on heparin previous to transfer. However, only $44(57.9 \%)$ patients reached therapeutic levels as measured by activated partial thromboplastin time (aPTT) prior to definitive revascularization. A delay of anticoagulation initiation $>48$ hrs from symptom onset was associated with increased 30-day reintervention rates compared with the $<6$ hrs group (66.7\% vs. $23.5 \%$; $p<0.05)$. However, time to reperfusion had no statistically significant impact on MALE, 30-day mortality, or 30-day interventional 
64 patency in our small cohorts. Additionally, patients with a previous revascularization had a higher 30-day 65 reintervention rate $(46.5 \% ; p<0.05)$.

66

\section{Conclusions:}

68

The practice of timely therapeutic anticoagulation of patients referred for ALI from community

69 facilities occurs less frequently than expected and is associated with an increased perioperative

70 reintervention rate. 
72

73

Paramount to the treatment of ALI is prompt diagnosis, anticoagulation, and timely revascularization to minimize the risk of limb loss. ${ }^{1}$ Unfortunately, delays in diagnosis and treatment continue to be a significant challenge, especially in those who are initially evaluated at a community hospital without dedicated vascular specialists. ${ }^{2}$ Our Level I Vascular Emergency program was initiated in 2009 as part of an effort to improve vascular outcomes in the State of Indiana. Transferring facilities state the diagnosis, hospital location, and urgency of pathology. All patients stable enough for transfer are accepted, and transportation is accomplished by ground or air at the discretion of the accepting physician and weather conditions.

Although revascularization strategies and outcomes are well studied, there is a relative dearth of knowledge regarding the effect of preintervention anticoagulation with timely triage and their effects on limb salvage and mortality. ${ }^{3}$ Therefore, the purpose of this study was to review our three-year experience, immediately after initiation of the Level I program, with the pretransfer care of ALI patients at our tertiary referral center and the outcomes associated with deviations from defined ideal management. 
Methods

89

After approval from the Indiana University Institutional Review Board (IRB), we performed a retrospective analysis of a prospectively maintained database of all patients diagnosed with ALI transferred to our facility via our Level I program from Jan 2010 to August 2013. For purposes of this study, ALI was defined as a cold, painful, ischemic limb presenting within a week of symptom onset. ${ }^{4,5}$

Patient demographics, comorbidities, Rutherford ischemia severity, time to heparinization, time to revascularization, and postoperative outcomes were abstracted via review of medical records. Times to emergency room (ER) presentation, heparin initiation, and revascularization were all recorded from the time of symptom onset and arbitrarily categorized into three subgroups ( $<6 \mathrm{hrs}, 6-48 \mathrm{hrs}$, and $>48 \mathrm{hrs})$. Our 30-day outcomes of interest were major amputation (above ankle), interventional patency, need for vascular reinterventions, and mortality. Categorical variables were compared with Fisher's exact tests while continuous variables ( \pm standard deviation) were compared with Student's T-tests at an $\alpha$ of 0.05 . 


\section{Results}

103

\section{Comorbidities}

In the three years queried, 103 patients with limb ischemia were transferred to us as a Level I Vascular Emergency; patients presenting with upper limb ischemia were excluded leaving 87 remaining cases. Demographics and comorbidities of the study population are detailed in Table 1. The average age of our population was $64.5( \pm 16.2)$ years with a slight predisposition towards the male gender $(57.5 \%)$. The most common comorbidity in this population was CAD (70.7\%). Atrial fibrillation, a frequent source of thromboembolism to the extremities, was present in $20.1 \%$ of subjects. Additionally, $39.0 \%$ of the patients had a previous history of ipsilateral vascular bypass and $40.2 \%$ were actively smoking at the time of ALI onset.

\section{Preinterventional Management}

The mean duration of time from symptom onset to initial outside hospital (OSH) evaluation was $18.3 \mathrm{hrs}$ (range $=1-118 \mathrm{hrs}$, median $=6 \mathrm{hrs}$ ). At the time of evaluation by the first physician, $53.8 \%$ had Rutherford class IIA ischemia and $36.3 \%$ had class IIB ischemia. The average delay from initial OSH evaluation to evaluation by a tertiary care hospital physician was $3.0 \mathrm{hrs}($ median $=2.0 \mathrm{hrs})$. After arrival to our facility from a mean transfer distance of 48.0 miles (range $1-183=$ miles, median $=47$ miles), mean delay until definitive blood flow restoration was $9.5 \mathrm{hrs}$ (median $=5.0 \mathrm{hrs}$ ).

Among the $76(87.4 \%)$ patients who received systemic heparin previous to transfer, only 44 $(57.9 \%)$ reached a documented therapeutic aPTT before their operation (Table 2). Of the remaining anticoagulated patients, 13 maintained nontherapeutic aPTTs while 19 patients did not have an aPTT measured before revascularization. Of those without a documented aPTT, $89.5 \%$ presented emergently 
for IIB ischemia and had intraoperative activated clotting times (ACT) on record. Of note, only 10 $(11.5 \%)$ patients received heparin and were documented therapeutic by aPTT in less than 6 hrs from symptom onset. Eleven (12.6\%) patients were not anticoagulated at all after presentation to the transferring facility. The reasons for not starting anticoagulation were unclear in all cases; however, two patients clearly did not have a salvageable limb. There were no differences between patients with IIA and IIB ischemia in terms of delays to heparin initiation $(p=0.25)$ or revascularization $(p=0.47)$.

\section{Intervention}

Arterial flow was restored in only three (3.4\%) patients via a completely percutaneous approach. All three patients were in IIA ischemia at presentation and consisted of one with an acutely thrombosed PTFE graft who was thrombolysed and two occlusions of the iliac and superficial femoral arteries treated by angioplasty and stenting. The remaining revascularizations utilized a primarily open technique. Of these patients, $50(59.5 \%)$ required primary thromboembolectomy while $28(33.3 \%)$ patients required a de novo lower extremity vascular bypass. Only $12.6 \%$ of the total study population was revascularized within 6 hrs of symptom onset. However, in the IIB ischemia subcohort, $36.3 \%$ was revascularized within 6 hrs of symptom onset.

\section{0-day Major Adverse Events}

\section{Major Amputations:}

The 30-day major amputation rate for all transferred ALI patients was $13.1 \%$ (Table 3). Time to anticoagulation did not affect amputation risk. We did not observe a difference between Rutherford IIA and IIB patients $(p=0.43)$; however, both groups experienced a lower amputation rate compared to those presenting with class III ischemia $(p<0.01)$. A history of an ipsilateral revascularization did not influence amputation risk $(p=0.49)$. Delays to therapeutic aPTT and revascularization did not influence perioperative major amputation rates in our population. 
155

156

157

158

159

160

161

162

163

164

165

166

167

168

169

170

171

172

173

174

175

176

177

178

179

\section{Reinterventions:}

The overall 30-day vascular reintervention rate was $36.8 \%$. We observed a difference in reintervention based on time to systemic heparinization with the $>48$ hrs cohort experiencing a $66.7 \%$ event rate compared to a $23.5 \%$ in patients heparinized in $<6 \mathrm{hrs}(p<0.05)$. Patients who had a previous ipsilateral revascularization were at a higher risk of reintervention $(46.7 \%$ vs $36.8 \%, p<0.05)$. No difference was observed between class IIA and IIB ischemia patients. Those who delayed ER presentation $(>48 \mathrm{hrs})$ also were at greater risk for perioperative reintervention $(p<0.05)$. Delays until therapeutic aPTT and revascularization did not influence perioperative vascular reintervention risk.

\section{Patency:}

The overall 30-day interventional patency rate was $72.2 \%$. There was no difference based on delay to heparinization; although, a nonsignificant inferior patency rate was noted in the $>48 \mathrm{hrs}$ cohort compared to the minimal delay cohort $(60 \%$ vs $76.5 \%, p=0.42)$. Additionally, we observed no difference between class IIA and IIB patients. There was also no difference based on delay to ER presentation or presence of previous ipsilateral bypass. Delays until therapeutic aPTT and revascularization did not influence perioperative patency.

\section{Mortality:}

The overall 30-day mortality was 7.9\%. The most common cause of death was septic shock $(n=3)$ followed by myocardial infarction $(n=1)$, multiorgan systems failure $(n=1)$, and acute mesenteric ischemia $(n=1)$. While there was no statistical difference in mortality by time to anticoagulation, a trend was observed. All patients who were heparinized without delay survived, compared to a $10.0 \%$ mortality $(p=0.28)$ in the $6-48$ hrs group and $13.3 \%(p=0.20)$ in the $>48$ hrs group. The degree of Rutherford ischemia did not seem to affect perioperative mortality in our population. Similarly, delays until therapeutic aPTT and revascularization did not influence perioperative mortality. 
In ALI, a sudden cessation of arterial blood flow directly blocks nerve, soft tissue, and

The latest ALI consensus practice guideline was developed in conjunction with the Society of Vascular Surgery, American Heart Association, Intersociety Consensus for the Management of Peripheral Arterial Disease, and representative bodies of cardiology, radiology, and vascular medicine. In these guidelines, only four level I recommendations pertain to the preoperative care of the ALI patient (Figure). ${ }^{10}$ At the time of presentation to any facility, patients suspected of ALI should be evaluated by an experienced clinician comfortable with the assessment of limb viability and can implement immediate medical therapy. At that time, a comprehensive history should be taken to determine the cause of thrombosis or embolization. Additionally, the initial evaluation should focus on the rapid assessment of 1) limb viability and 2) potential for salvage. It is key to note that this assessment does not require imaging, especially if it may delay appropriate triage. After diagnosis, prompt systemic anticoagulation should be initiated unless a hard contraindication is present. ${ }^{11}$

The use of any antiplatelet and/or anticoagulant must be communicated clearly and concisely by the transferring team. Especially important details to include are time of medication initiation and dose. In our review, 13 patients received heparin without documentation of initiation time by the transferring 
team. Additionally, 11 patients did not receive heparin at the transferring facility at all. While two limbs were clearly nonsalvageable, none of these 11 patients had a clear contraindication on retrospective chart review. While a possible explanation may be an attempt to avoid coagulopathy during surgery by clinicians uncomfortable with preoperative anticoagulation, the short half-life of unfractionated heparin makes cessation of medication administration on the way to the OR an ideal therapeutic plan. ${ }^{12} \mathrm{We}$ observed a trend of increasing mortality in those who had a delay until systemic heparinization. This effect may prove to be significant with a higher-powered study. Failure to anticoagulate in the ALI population is not an isolated problem for our facility; an European experience reported by Spanos et al reported their four-year experience of 112 consecutive ALI patients and found that only $67 \%$ of them were anticoagulated at the time of arrival after transfer. ${ }^{13}$ A concerted effort should be made to emphasize the importance of prompt anticoagulation to our transferring colleagues.

The superior outcomes observed in ALI patients who received timely anticoagulation occurred regardless of their aPTT status in our study, thus underscoring the importance of anticoagulation and less so the emphasis on clotting times in limb salvage. We did not observe a significant difference in outcomes with regards to perioperative mortality, patency, and major amputation in those who had a $>48$ hr delay to heparinization. However, this was almost certainly due to lack of power as clinical experience would dictate a morbid clinical course in these patients. Unless contraindicated, the best anticoagulant in the acute setting is IV unfractionated heparin. ${ }^{14}$ The dosing of which is discussed in detail elsewhere. ${ }^{15}$ Despite limitations, aPTT remains the most convenient and frequently used method for monitoring in vivo heparin response. It should be measured minutes after initial medication bolus to confirm therapeutic effect and continuously monitored to gauge needed changes to the continuous rate. ${ }^{16}$

A problem we encountered in our cohort was a significant delay in presentation to the initial hospital of more than 18 hours from symptom onset. The worst offenders were those with baseline disease and therefore assumed acute limb pain was a day-to-day variant of symptoms and not a sudden 
progression of disease. This incorrect assumption often made a difference between the salvage of a functional limb or not. Therefore, the burden is on us, as vascular surgeons, to educate our patients on signs of ischemic progression from chronic disease to ALI in the office setting before it occurs.

Seven patients $(8 \%)$ were transferred without documentation of a lower extremity pulse exam in the history and physical of the initial evaluating physician. Not only is this a clinical concern, but it may have serious medicolegal ramifications. A detailed lower extremity vascular exam is standard of care for any patient with limb pain, sensory loss, or motor dysfunction. ${ }^{17}$ Although the pulse exam may be subjective, the following classification is recommended at minimum: a) normal, b) weakly palpable, c) doppler signal present, and d) no signal. ${ }^{18}$ The status of the dorsalis pedis and posterior tibial arteries on both sides need to be documented in the medical record.

As is the case for any retrospective review, this study has some inherent limitations. ${ }^{19}$ However, a recurring deficit which developed during data collection was the incomplete documentation from the transferring facilities on time of symptom onset, degree of ischemia, and time and effect of anticoagulation despite receiving all available medical records. Consequently, the patient pool for statistical analysis was reduced affecting the power to detect outcome differences. Although we did receive direct transfers from community vascular surgeons, most of the patients arrived from ERs staffed by emergency, family, and internal medicine specialists often uncomfortable with ALI management. As such, it is of upmost importance that the accepting physician clearly state to the transferring physician the treatment plan before, during, and after the transfer process. 
ALI is a challenging pathology for the vascular surgeon. In many regions, referral of those

256 diagnosed at community hospitals to tertiary centers have become the established system. In this study, we report inferior perioperative outcomes associated with patients who did not receive treatment per ALI

258 practice guidelines. Therefore, improved system-wide protocols in the diagnosis, care, and transfer of 259 these patients are needed to provide safe, timely care. 
1. Wang JC, Kim AH, Kashyap VS. Open surgical or endovascular revascularization for acute limb ischemia. Journal of vascular surgery. 2016;63(1):270-278.

2. Lukasiewicz A. Treatment of acute lower limb ischaemia. VASA. Zeitschrift fur Gefasskrankheiten. 2016;45(3):213-221.

3. Lambert MA, Belch JJ. Medical management of critical limb ischaemia: where do we stand today? Journal of internal medicine. 2013;274(4):295-307.

4. Creager MA, Belkin M, Bluth EI, Casey DE, Jr., Chaturvedi S, Dake MD, et al. 2012 ACCF/AHA/ACR/SCAI/SIR/STS/SVM/SVN/SVS key data elements and definitions for peripheral atherosclerotic vascular disease: a report of the American College of Cardiology Foundation/American Heart Association Task Force on Clinical Data Standards (Writing Committee to Develop Clinical Data Standards for Peripheral Atherosclerotic Vascular Disease). Circulation. 2012;125(2):395-467.

5. Rutherford RB, Baker JD, Ernst C, Johnston KW, Porter JM, Ahn S, et al. Recommended standards for reports dealing with lower extremity ischemia: revised version. Journal of vascular surgery. 1997;26(3):517-538.

6. de Franciscis S, De Caridi G, Massara M, Spinelli F, Gallelli L, Buffone G, et al. Biomarkers in post-reperfusion syndrome after acute lower limb ischaemia. International wound journal. 2016;13(5):854-859.

7. Hausenloy DJ, Yellon DM. Ischaemic conditioning and reperfusion injury. Nature reviews. Cardiology. 2016;13(4):193-209.

8. Falluji N, Mukherjee D. Critical and acute limb ischemia: an overview. Angiology. 2014;65(2):137-146.

9. Hardman RL, Jazaeri O, Yi J, Smith M, Gupta R. Overview of Classification Systems in Peripheral Artery Disease. Seminars in Interventional Radiology. 2014;31(4):378-388.

10. Gerhard-Herman MD, Gornik HL, Barrett C, Barshes NR, Corriere MA, Drachman DE, et al. 2016 AHA/ACC Guideline on the Management of Patients with Lower Extremity Peripheral Artery Disease: Executive Summary. Vascular medicine (London, England). 2017;22(3):Np1np43.

11. Kalathottukaren MT, Haynes CA, Kizhakkedathu JN. Approaches to prevent bleeding associated with anticoagulants: current status and recent developments. Drug delivery and translational research. 2017.

12. van Rein N, Biedermann JS, van der Meer FJM, Cannegieter SC, Wiersma N, Vermaas HW, et al. Major bleeding risks of different low-molecular-weight heparin agents: a cohort study in 12 934 patients treated for acute venous thrombosis. Journal of thrombosis and haemostasis : JTH. 2017;15(7):1386-1391.

13. Spanos K, Athanasoulas A, Argyriou C, Vassilopoulos I, Giannoukas AD. Acute limb ischemia and anticoagulation in patients with history of atrial fibrillation. International angiology : a journal of the International Union of Angiology. 2016;35(5):510-515.

14. Kearon C, Akl EA, Ornelas J, Blaivas A, Jimenez D, Bounameaux H, et al. Antithrombotic Therapy for VTE Disease: CHEST Guideline and Expert Panel Report. Chest. 2016;149(2):315352.

15. Safani M, Tobias S, Robinson M, Hwang J, Thomas GS. Average-Weight Methodology in Weight-Based Unfractionated Heparin Therapy in the Presence of Obesity. Chest. 2017;151(5):1187-1188.

16. Hosch LM, Breedlove EY, Scono LE, Knoderer CA. Evaluation of an Unfractionated Heparin Pharmacy Dosing Protocol for the Treatment of Venous Thromboembolism in Nonobese, Obese, and Severely Obese Patients. The Annals of pharmacotherapy. 2017;51(9):768-773.

17. Vemulapalli S, Patel MR, Jones WS. Limb ischemia: cardiovascular diagnosis and management from head to toe. Current cardiology reports. 2015;17(7):611. 
310

311

312

313

18. Montorfano MA, Pla F, Vera L, Cardillo O, Nigra SG, Montorfano LM. Point-of-care ultrasound and Doppler ultrasound evaluation of vascular injuries in penetrating and blunt trauma. Critical ultrasound journal. 2017;9(1):5.

19. Mantel N. Synthetic retrospective studies and related topics. Biometrics. 1973;29(3):479-486. 


\section{Legends}

Table 1

Demographics and comorbidities

\section{Table 2}

Delays in treatment

\section{Table 3}

Perioperative outcomes by delay until heparinization from symptom onset. Fisher's exact testing was performed comparing the minimally delayed cohort ( $<6 \mathrm{hrs})$ to the remaining groups.

\section{Figure}

Summary of the latest AHA/ACC consensus practice guidelines 


\begin{tabular}{|l|c|}
\hline \multicolumn{1}{|c|}{ Condition } & Incidence \\
\hline Age & $64.5 \pm 16.2$ \\
\hline Male & $57.5 \%$ \\
\hline CAD & $70.7 \%$ \\
\hline HTN & $67.9 \%$ \\
\hline Active Smoker & $40.2 \%$ \\
\hline HLD & $37.8 \%$ \\
\hline DM & $32.1 \%$ \\
\hline AF & $20.1 \%$ \\
\hline COPD & $18.3 \%$ \\
\hline Previous Ipsilateral Revascularization & $39.0 \%$ \\
\hline Rutherford Classification at Presentation & Incidence \\
\hline I & $7.5 \%$ \\
\hline IIA & $53.8 \%$ \\
\hline IIB & $36.3 \%$ \\
\hline III & $2.5 \%$ \\
\hline
\end{tabular}




\section{Delay to ER Presentation ( $\mathrm{n=87}$ ) Incidence}

\begin{tabular}{l|l|l|}
\hline$<6$ hrs & $37.9 \%$ \\
\hline
\end{tabular}

\begin{tabular}{ll}
$6-48 \mathrm{hrs}$ & $25.3 \%$ \\
\hline
\end{tabular}

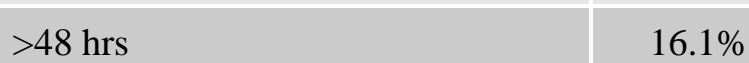

\begin{tabular}{l|l}
\hline Unknown & $20.7 \%$ \\
\hline
\end{tabular}

\begin{tabular}{|l|r|}
\multicolumn{1}{|c|}{ Delay to Heparin $(\mathbf{n = 7 6})$} & Incidence \\
\hline$<6 \mathrm{hrs}$ & $23.7 \%$ \\
\hline $6-48 \mathrm{hrs}$ & $39.5 \%$ \\
\hline$>48 \mathrm{hrs}$ & $19.7 \%$ \\
\hline Unknown & $17.1 \%$ \\
\hline Delay to Revascularization $(\mathbf{n = 7 1})$ & Incidence \\
\hline$<6 \mathrm{hrs}$ & $12.7 \%$ \\
\hline $6-48 \mathrm{hrs}$ & $69.0 \%$ \\
\hline$>48 \mathrm{hrs}$ & $18.3 \%$ \\
\hline
\end{tabular}


Delay Until Systemic Heparinization

\begin{tabular}{|l|c|c|c|}
\hline & $<\mathbf{6 h r}$ & $\mathbf{6 - 4 8 h r}$ & $\mathbf{4 8 h r}$ \\
\hline 30-day Amputation & $\mathbf{1 6 . 7 \%}$ & $\mathbf{6 . 9 \%}$ & $\mathbf{2 1 . 4 \%}$ \\
\hline 30-day Reintervention & & $p=0.36$ & $p=1.0$ \\
\hline & $\mathbf{2 3 . 5 \%}$ & $\mathbf{3 2 . 2 \%}$ & $\mathbf{6 6 . 7 \%}$ \\
\hline $\mathbf{3 0 - d a y}$ Mortality & & $p=0.74$ & $\mathbf{p = 0 3}$ \\
\hline & $\mathbf{0 \%}$ & $\mathbf{1 0 . 0 \%}$ & $\mathbf{1 3 . 3 \%}$ \\
\hline 30-day Patency & $\mathbf{7 6 . 5 \%}$ & $p=0.28$ & $p=0.20$ \\
\hline
\end{tabular}




\section{Patient Suspected of ALI}

Tenet 1

Patients suspected of ALI should be emergently evaluated by a clinician with sufficient experience to determine limb viability and implement appropriate therapy
Tenet 2

A comprehensive history should be obtained to

determine the cause of

thrombosis and/or embolization
Tenet 3

The initial evaluation should determine 1) limb viability and

2) potential for salvage - it does not require imaging
Tenet 4

Systemic anticoagulation should be initiated immediately, unless a hard contraindication is present 